\title{
Piptadenia (Leguminosae, Mimosoideae) in the state of Minas Gerais, Brazil
}

\author{
Mariana Neves Moura ${ }^{1,3}$, Vanessa Terra ${ }^{1,2}$ \& Flávia C.P. Garcia ${ }^{1}$
}

\begin{abstract}
The genus Piptadenia includes approximately 24 species that are distributed in the tropical regions of South America, with centers of diversity in the Amazon and Atlantic Forest in Brazil in which twentyone species occur. A taxonomic study of Piptadenia was conducted in the state of Minas Gerais based on an analysis of approximately 270 specimens from 11 herbaria, field observations and field work in the main conservation units and other focal areas in Minas Gerais. These areas include the three vegetation domains in the state: Atlantic Forest, Cerrado and Caatinga. Six of the species in the genus were sampled: $P$. adiantoides, $P$. gonoacantha, $P$. macradenia, $P$. micracantha, $P$. paniculata and $P$. viridiflora. Two species that have previously been cited in the state were not found in this study: $P$. irwinii and $P$. stipulacea. These species were not found in the herbaria collections that were consulted or during the field work. We present an identification key for the Minas Gerais species of Piptadenia as well as descriptions, illustrations, geographical distributions, comments about the taxonomic characters and some information related to the flowering and fruiting periods.
\end{abstract}

Key words: floristics, morphology, taxonomy, phytogeographic domains.

\section{Resumo}

O gênero Piptadenia apresenta cerca de 24 espécies que estão distribuídas na região tropical da América do Sul, com centros de diversidade na Amazônia e na Floresta Atlântica do Brasil, que abriga vinte e uma espécies deste gênero. Os estudos taxonômicos de Piptadenia foram conduzidos no estado de Minas Gerais, baseados na análise de 270 espécimes de 11 herbários, observações de campo e coletas de material botânico nas principais Unidades de Conservação de Minas Gerais, abrangendo os três biomas presentes no Estado: Floresta Atlântica, Cerrado e Caatinga. Seis espécies do gênero em estudo foram amostradas: Piptadenia adiantoides, $P$. gonoacantha, $P$. macradenia, $P$. micracantha, $P$. paniculata e $P$. viridiflora. Duas espécies foram citadas para o Estado, mas não constam neste trabalho: Piptadenia irwinii e $P$. stipulacea, visto que não apresentaram nenhum material testemunho nos acervos dos herbários consultados e não foram encontradas nos trabalhos de campo. Neste trabalho apresentamos uma chave de identificação para as espécies de Piptadenia que ocorrem em Minas Gerais, descrições, ilustrações, distribuição geográfica e comentários sobre os caracteres taxonômicos, bem como algumas informações relacionadas aos períodos de floração e frutificação de cada espécie.

Palavras-chave: florística, morfologia, taxonomia, domínios fitogeográficos.

\section{Introduction}

Piptadenia Benth. belongs to Leguminosae Jussieu, the third largest family of flowering plants, which has a cosmopolitan distribution and comprises 36 tribes, 727 genera and approximately 19,000 species (Lewis et al. 2005). The genus Piptadenia includes approximately 24 species that are distributed in the tropical regions of South America with centers of diversity in the Amazon and Atlantic Forest in Brazil (Luckow 2005) in which 21 species occur (Morim 2010; BFG 2015). Until now, nine species have been reported in Minas Gerais State (Tamashiro 1989; Oliveira-Filho 2006; Morim 2010).

\footnotetext{
${ }^{1}$ Universidade Federal de Viçosa, Department of Plant Biology, Ph Rolfs s/n, Centro, 36570-000, Viçosa, MG, Brazil.

${ }^{2}$ Universidade Federal de Uberlândia, Inst. Ciências Agrárias, Rod. LMG 746, Km 1, s/n, bl. 1a, 38500-000, Monte Carmelo, MG, Brazil.

${ }^{3}$ Author for correspondence: mnevesmoura@gmail.com
} 
Phylogenetic studies have indicated a greater affinity of Piptadenia with Parapiptadenia Brenan, Pseudopiptadenia Rauschert and Plathymenia Benth. as all share spicate raceme inflorescences and anthers with glands (Grimes 1999; Luckow 2005). Molecular data have shown that Piptadenia is a polyphyletic genus (Luckow et al. 2003; Jobson \& Luckow 2007), and as a result of these molecular studies, two species were transferred to Pityrocarpa (Benth.) Britton \& Rose: Piptadenia moniliformis Benth. and Piptadenia obliqua (Pers.) J.F. Macbride (Jobson \& Luckow 2007).

Tamashiro (1989), in a taxonomic study of Piptadenia species in southeast Brazil, found four species in Minas Gerais, but the sampling effort in the state was not exhaustive, and taxonomic changes have since occurred in the genus. OliveiraFilho (2006) cited seven species as occurring in the state in a floristic list that was assembled without a taxonomic approach and that only considered trees. Finally, Morim (2010), in the List of Flora Species in Brazil, reported the occurrence of six species in Minas Gerais. However, none of these studies involved collection, revision and characterization with sampling specifically for Piptadenia.

Thus, since the above studies do not present a consensus on this issue, the main goal of this study was to understand the diversity of Piptadenia in Minas Gerais and to confirm the number of species occurring in the state through increased field sampling, morphological characterization and the review of herbaria collections.

\section{Material and Methods}

The study was based on an analysis of approximately 270 specimens from 11 herbaria: BHZB, CEN, CESJ, HUFU, HXBH, IBGE, IBTSP, OUPR, PAMG, RB, UB, VIC (abbreviations follow Holmgreen \& Holmgreen [2009]), field observations and collections of botanical material from the main conservation units and specific areas in Minas Gerais, including the three vegetation domains present in the state: Atlantic Forest, Cerrado and Caatinga (Drummond et al. 2005). From the RB, the "Reflora- Virtual Herbarium" website was consulted and all the images observed are cited as "RB!" in the examined material.

A total of 27 expeditions to collect botanical specimens were undertaken between January 2008 and August 2010 in 47 conservation areas in the state of Minas Gerais, which are mainly distributed throughout state and national parks as well as biological, ecological and sustainable reserves (Tab. 1; Fig. 1). The expeditions also included 14

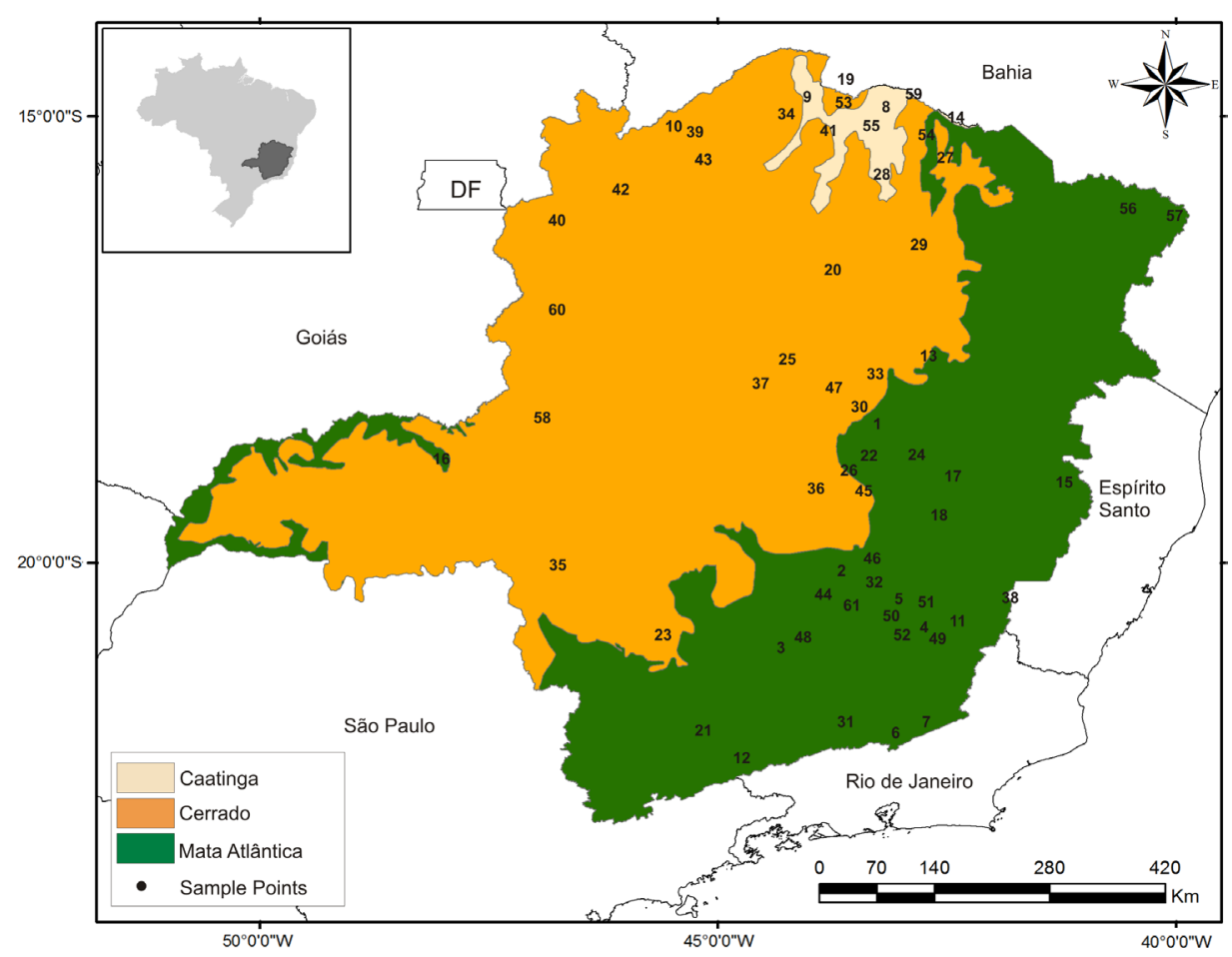

Figure 1 - State of Minas Gerais with phytogeographical areas and sampling sites (Fernandes 2011). 
Table 1 -Conservation Units sampled in Minas Gerais, Brazil. $N^{\circ} .=$ number of the Conservation Units corresponding in Figure 1.

\begin{tabular}{|c|c|c|}
\hline No. & Conservation Units & Cities \\
\hline 1 & Área de Proteção Ambiental Água das Vertentes & $\begin{array}{l}\text { Couto de Magalhães de Minas, Diamantina, Felício dos Santos, Rio } \\
\text { Vermelho, Santo Antônio do Itambé, Serra Azul de Minas e Serro }\end{array}$ \\
\hline 2 & $\begin{array}{l}\text { Área de Proteção Ambiental da Cachoeira das } \\
\text { Andorinhas }\end{array}$ & Ouro Preto \\
\hline 3 & Área de Proteção Ambiental da Serra de São João & $\begin{array}{l}\text { Cel. Xavier Chaves, Prados, Santa Cruz de Minas, São João Del } \\
\text { Rei e Tiradentes }\end{array}$ \\
\hline 4 & Área de Proteção Ambiental Nô da Silva & Cajuri \\
\hline 5 & $\begin{array}{l}\text { Estação de Pesquisa, Treinamento e Educação } \\
\text { Ambiental Mata do Paraíso }\end{array}$ & Viçosa \\
\hline 6 & Estação Ecológica Mar de Espanha & Mar de Espanha \\
\hline 7 & Floresta Estadual Uaimii & Ouro Preto \\
\hline 8 & Parque Estadual Caminho das Gerais & Mamonas, Monte Azul e Gameleiras \\
\hline 9 & Parque Estadual da Mata Seca & Manga \\
\hline 10 & Parque Estadual da Serra das Araras & Chapada Gaúcha \\
\hline 11 & Parque Estadual da Serra do Brigadeiro & $\begin{array}{l}\text { Araponga, Fervedouro, Miradouro, Ervália, Sericita, Pedra Bonita, } \\
\text { Muriaé e Divino }\end{array}$ \\
\hline 12 & Parque Estadual da Serra do Papagaio & Aiuruoca, Alagoa, Baependi, Itamonte e Pouso Alto \\
\hline 13 & Parque Estadual da Serra Negra & Itamarandiba \\
\hline 14 & Parque Estadual de Montezuma & Montezuma \\
\hline 15 & Parque Estadual de Sete Salões & Resplendor, Santa Rita do Itueto, Conselheiro Pena e Itueta \\
\hline 16 & Parque Estadual do Pau Furado & Uberlândia e Araguari \\
\hline 17 & Parque Estadual do Rio Corrente & Açucena \\
\hline 18 & Parque Estadual do Rio Doce & Marliéria, Dionísio e Timóteo \\
\hline 19 & Parque Estadual do Verde Grande & Matias Cardoso \\
\hline 20 & Parque Estadual Lapa Grande & Montes Claros \\
\hline 21 & Parque Estadual Nova Baden & Lambari \\
\hline 22 & Parque Estadual Pico do Itambé & Santo Antônio do Itambé, Serro e Serra Azul de Minas \\
\hline 23 & Parque Estadual Serra da Boa Esperança & Serra da Boa Esperança \\
\hline 24 & Parque Estadual Serra da Candonga & Guanhães \\
\hline 25 & Parque Estadual Serra do Cabral & Buenópolis, Joaquim Felício \\
\hline 26 & Parque Estadual Serra do Intendente & Conceição do Mato Dentro \\
\hline 27 & Parque Estadual Serra Nova & Rio Pardo de Minas \\
\hline 28 & Parque Estadual Veredas do Peruaçu & Januária \\
\hline 29 & Parque Estadual de Grão-Mogol & Grão-Mogol \\
\hline 30 & Parque Estadual do Biribiri & Diamantina \\
\hline 31 & Parque Estadual do Ibitipoca & Lima Duarte e Santa Rita do Ibitipoca \\
\hline 32 & Parque Estadual do Itacolomi & Ouro Preto e Mariana \\
\hline 33 & Parque Estadual do Rio Preto & São Gonçalo do Rio Preto \\
\hline 34 & Parque Nacional Cavernas do Peruaçu & Januária, São João das Missões, Itacarambi, \\
\hline 35 & Parque Nacional da Serra da Canastra & São Roque de Minas, Sacramento e Delfinópolis \\
\hline 36 & Parque Nacional da Serra do Cipó & $\begin{array}{l}\text { Jaboticatubas, Santana do Riacho, Morro do Pilar e Conceição do } \\
\text { Mato Dentro }\end{array}$ \\
\hline
\end{tabular}




\begin{tabular}{|c|c|c|}
\hline $\mathbf{N}^{0}$. & Conservation Units & Cities \\
\hline 37 & Parque Nacional das Sempre Vivas & Bocaiúva, Buenópolis, Diamantina e Olhos d’Água \\
\hline 38 & Parque Nacional do Caparaó & Alto Caparaó, Caparaó, Alto Jequitibá e Espera Feliz \\
\hline 39 & Parque Nacional Grande Sertão Veredas & Chapada Gaúcha \\
\hline 40 & Reserva Ambiental de Cabeceira Grande & Cabeceira Grande \\
\hline 41 & Reserva Biológica Serra Azul & Jaíba \\
\hline 42 & Reserva Ecológica de Sagarana & Arinos \\
\hline 43 & $\begin{array}{l}\text { Reserva Estadual de Desenvolvimento } \\
\text { Sustentável Veredas do Acari }\end{array}$ & Chapada Gaúcha \\
\hline 44 & $\begin{array}{l}\text { Reserva Particular do Patrimônio Natural Luiz } \\
\text { Carlos Jurovsk Tamassia }\end{array}$ & Ouro Branco \\
\hline 45 & $\begin{array}{l}\text { Reserva Particular do Patrimônio Natural Reserva } \\
\text { da Cachoeira }\end{array}$ & Santana do Riacho \\
\hline 46 & $\begin{array}{l}\text { Reserva Particular do Patrimônio Natural } \\
\text { Santuário do Caraça* }\end{array}$ & Catas Altas e Santa Bárbara \\
\hline 47 & $\begin{array}{l}\text { Reserva Particular do Patrimônio Natural Serafim } \\
\text { Melo Jardim }\end{array}$ & Diamantina \\
\hline
\end{tabular}

target areas in the state, particularly micro-regions without protected areas (Tab. 2; Fig. 1). Field collection was performed to increase the sampling effort and provide new specimens for comparison.

Morphological analyses were only performed on specimens from Minas Gerais State and included measures of the vegetative structures in dehydrated material while the floral structures were measured after rehydration. Illustrations were constructed with the aid of a stereomicroscope using botanical samples herborized and/or fixed in $70 \%$ ethanol. The abbreviations shown in the descriptions, taxonomic remarks and lists of examined materials are as follows: $\mathrm{m}=$ meter, $\mathrm{mm}=$ millimeter, $\mathrm{fl}=$ flowering and $\mathrm{fr}=$ fruiting.

Identification was performed by morphological analysis based on the taxonomic literature for the genus (Bentham 1840; Bentham

Table 2 - Specific areas sampled in Minas Gerais, Brazil. $\mathrm{N}^{\mathrm{o}}=$ number of the specific areas corresponding in Figure 1.

\begin{tabular}{lll}
\hline$N^{0}$. & Specific areas sampled & Cities \\
\hline 48 & Fazenda Água Limpa & Prados \\
49 & Fazenda Monte Libra & Cajuri \\
50 & Mata da Biologia & Campus/UFV \\
51 & Mata da Silvicultura & Campus/UFV \\
52 & Mata do Seu Nico & Viçosa \\
53 & Perímetro urbano & Matias Cardoso \\
54 & Perímetro urbano & Mato Verde \\
55 & Perímetro urbano & Jaíba \\
56 & Perímetro urbano & Almenara \\
57 & Perímetro urbano & Santa Maria do Salto \\
58 & Perímetro urbano & Coromandel \\
59 & Perímetro urbano & Mamonas \\
60 & Região do machadinho & Paracatu \\
61 & Serra da Capanema & Ouro Preto \\
\hline
\end{tabular}


1875; Tamashiro 1989; Lewis et al. 2005; Luckow 2005) and by consulting the collections of the herbaria cited above. The analytical and diagnostic characteristics of the studied species were illustrated.

The geographical distributions were based on information from the literature (Tamashiro 1989; Morim 2010) and herbaria records. Phytogeographic information was obtained from the herbaria records when available; the phytophysiognomies were adapted from Veloso et al. (1991) and IBGE (1992), and the phytogeographic areas were adapted from Ab'Saber (2003). Flowering and fruiting periods were obtained from the herbaria records and from observations during the field work.

The maps of geographical distributions were elaborated using ArcGIS 10.1 software.

\section{Results and Discussion}

1. Piptadenia Benth., J. Bot. (Hooker) 2(11): 135. 1840.

Scandent shrubs, lianas or trees up to $25 \mathrm{~m}$ tall. Branches cylindrical, unarmed or armed, with straight or curved aculeus. Stipules deciduous or, when persistent, modified in aculeus. Leaves bipinnate, petiolate; petiole cylindrical; leaf rachis cylindrical, unarmed or armed; petiolar nectaries sessile, patelliform, cupuliform or verruciform. Pinnae opposite. Leaflets linear, ovate, ovatelanceolate or obovate, glabrous or pilous. Basic inflorescence unit is spiciform, isolated or in panicles, axillary or terminal. Flowers hermaphrodite, actinomorphic, sessile; calyx pentamerous, campanulate, glabrous, sericeous or tomentose, gamosepalous; corolla pentamerous, gamopetalous at the base, campanulate, glabrous or sericeous; stamens 10, free at the base; anthers with stipitate gland at the connective, deciduous in anthesis; ovary stipitate, unicarpellate, pluriovulate, glabrous, tomentose or villous. Legumes flat, linear, glabrous; seeds numerous, oblong, rounded or obovate; pleurograma present.

Comments: Six species of the genus were found in Minas Gerais: Piptadenia adiantoides (Spreng.) J.F. Macbride, P. gonoacantha (Mart.) J.F. Macbride, P. macradenia Benth., P. micracantha Benth., P. paniculata Benth. and $P$. viridiflora (Kunth) Benth. The species may be present as lianas or shrubs, which are predominantly trees, in the state. No species of Piptadenia are considered endangered in Minas Gerais or in Brazil according to the List of Endangered Brazilian Species MMA (2014).

\section{Identification key for the taxa of the genus Piptadenia Benth. in the state of Minas Gerais}

1. Plants with persistent stipules transformed into aculeus. 1.6. Piptadenia viridiflora

1'. Plants with deciduous stipules, never turned into aculeus

2. Leaves with 3-7 pairs of pinnae; oval-lanceolate leaflets, ovate or obovate-elliptical, with a central midrib, with an obtuse or oblique base, $13-30 \times 6-13 \mathrm{~mm}$

3. Armed rachis; leaflets 3-5 pairs per pinna, ovate or obovate-elliptical, base oblique, apex obtuse; petiolar nectary verruciform, oblong; spiciform axillary inflorescence with 1-2 spikes per axilla; calyx glabrous 1.1. Piptadenia adiantoides

3'. Rachis unarmed; leaflets 7-15 pairs per pinna, oval-lanceolate, obtuse base, acute apex; nectary petiolar patelliform; spiciform inflorescence grouped in panicles; calyx sericeous .. 1.5. Piptadenia paniculata

2'. Leaves with 7-14 pairs of pinnae; linear leaflets with an eccentric main vein, with a truncated or cordate base, $3-8 \times 0.5-1.6 \mathrm{~mm}$

4. Unarmed branches; rachis unarmed; inflorescence grouped in panicles; calyx tomentose; corolla sericeous 1.3. Piptadenia macradenia

4'. Armed branches; armed rachis; spiciform axillary inflorescences; calyx glabrous or sericeous; corolla glabrous

5. Scandent shrubs to climbers; branches not winged; petiolar nectary verruciform, oblong; calyx glabrous; ovary tomentose. 1.4. Piptadenia micracantha

5'. Trees; winged branches; petiolar nectary cupuliform; calyx sericeous; ovary glabrous 1.2. Piptadenia gonoacantha 
1.1. Piptadenia adiantoides (Spreng.) J.F. Macbr. Contributions from the Gray Herbarium of Harvard University 59: 17. 1919.

Fig. 2a-d

Scandent shrub or, rarely, small tree of ca. 7 $\mathrm{m}$; branches cylindrical, unarmed or armed, curved aculeus, 1.9-3 mm long. Stipules deciduous, not observed. Leaves with 3-4 pairs of pinnae; leaf rachis $5-15 \mathrm{~cm}$ long, cylindrical with curved aculeus; leaflets ovate or obovate-elliptical, 3-5 pairs per pinna, $13-22 \times 6-12 \mathrm{~mm}$, glabrous on both surfaces, rarely sericeous, base oblique, apex obtuse, mucronate, central midrib; petiolar nectary verruciform, oblong. Inflorescences spiciform, 1-2 per axil, 6-10 cm long. Calyx, 0.89-1.2 mm long, glabrous; corolla 1.61-1.97 mm long, glabrous; filaments roseate or vinaceous; ovary stipitate, tomentose. Legume 11-13 $\times 2.2-2.7 \mathrm{~cm}$; seed oblong, $0.8-1.2 \times 5.2-5.5 \mathrm{~mm}$.

This species is endemic to Brazil. It occurs in the states of Paraíba, Pernambuco, Bahia, Minas Gerais, Espírito Santo, São Paulo, Rio de Janeiro and Paraná (BFG 2015). It is found from the southeast to the northern regions of Minas Gerais (Fig. 3) in Cerrado, Caatinga and Atlantic Forest domains.

Piptadenia adiantoides is easily recognized by its large leaflets compared to the other species. The leaflets are ovate or obovate-elliptical, and the petiolar nectary is verruciform, oblong, and located just below the first pair of pinnae. The branches have curved aculeus, and the shape of the nectary resembles $P$. micracantha, which differs in the shape, size and quantity of leaflets per pinna ( $P$. micracantha has 32-50 pairs of pinnae per leaflet vs. $P$. adiantoides with 3-4 pairs of pinnae per leaflet). Finally, $P$. adiantoides occurs in all three domains of the state, but $P$. micracantha is restricted to the Atlantic Forest domain.

Examined material: Belo Horizonte, Praça Nacional, Portaria, 4.VI.2008, fl. e fr., J. Ordones et al. 921 (BHZB); 23.III.1919, fr., A. Gehrt 162 (photo RB!); 17.VI.1934, H.L. Mello Barreto 6453 (BHCB); 13.V.1980, J.A. Oliveira (BHCB 869); 1.VI.1986, T.S.M. Grandi (BHCB 10619); 21.VII.1992, J. Pires (BHCB 19659); 11.IV.1995, J.A. Lombardi 738 (BHCB); 10.VIII.1995, J.A. Lombardi \& L.G. Temponi 920 (BHCB); 29.V.1996, J.D. Souza 91 (BHCB); Berilo, 13.II.2001, E. Tameirão Neto 3276 (BHCB); Caratinga, Estação Biológica de Caratinga, estrada do Nilo, 23.VII.1984, fr., E.M. Andrade, 289 (BHCB, photo RB!); Estação Biológica de Caratinga, 22.V.1984, fl., P.M. Andrade 166 (photo RB!); 9.V.1889, L.V. Costa \& P.H.A. Pequeno 248 (BHCB 16397); 20.XI.1984; M.A. Lopes et al. 511 (BHCB); XII.1990, C.V. Mendonça
Filho 127 (BHCB); Catas Altas, Caraça, trilha até Tabões, fr., J. Ordones et al. 920 (BHZB); 1.IV.2000, R.C. da Mota 733 (BHCB); 18.VI.2002, T.M.A. Alves \& M. Sobral 53 (BHCB); Catuji, 13.V.1983, fl., Hatschbach, G. 46303 (photo RB!); Conceição do Mato Dentro, Pousada Vale das Pedras 1.VII.2008, fr., L.H.Y Kamino 1021 (photo RB!), 1.VII.2008, fl., L.H.Y Kamino 1026 (photo RB!); Coronel Pacheco, Estação Experimental de Café, 20.V.1941, fl., E.P. Heringer 601 (photo RB!); Descoberto, Reserva Biológica da Represa do Grama, 15.VI.2002, fr., R.C. Forzza, B.K.S. Franco, L.D. Meireles 2202 (CESJ); Diamantina, Serra do espinhaço, 17.III.1970, fl., H.S. Irwin et al. 27772 (UB); Itabira, 1.IV.2002, fl., J.R. Stehmann 3076 (CESJ); Governador Valadares, caminho para Teófilo Otoni, BR 116, 24.IX.1999, fr., S.M. Faria 1785 (photo RB!); Itabira, 1.IV.2002, J.R. Stehmann 3076 (BHCB); Itabirito, 25.III.1994, fl., W.A. Teixeira,(BHCB 24263); Itamonte, Fazenda Campo Redondo, s.d., F.M. de B. Pereira 63 (photo RB!); Hotel Casa Alpina, Est. subindo para as antenas, 4.VIII.2011, fr., T.A. Batista 415 (photo RB!); Jaboticatubas, $\mathrm{Km} 126$ ao longo da rodovia Lagoa Santa-Conceição do mato dentro, 7.IV.1974, fl., J. Semir, M. Sazima 145026 (IBTSP); Km 127 ao longo da rodovia Lagoa Santa - Conceição do Mato Dentro - Diamantina, 31.III.1980, fl., Furlan, A. 6093 (photo RB!); Juiz de Fora, Morro do Imperador, 8.VII.2001, fr., D.S. Pifano \& A.S.M. Valente 33996 (CESJ); Manga, Jaíba, 5.IX.1974, fr., G.M. Magalhães, M.B. Ferreira 3040 (PAMG); Mariana, Cibrão, 23.III.2004, f1., M.C.T.B. Messias 868 (OURP); Linha Catódica, Sentido- Samarco, 25.VIII.1999, fr., S.M. de Faria 1758 (photo RB!); Marliéria, 16.VII.1996, J.A. Lombardi 1295 (BHCB); Ouro Preto, 29.III.2005, fl., L.C.P. Lima 310 \& E.D. Silva (VIC); Parque Estadual do Itacolomi, morro do cachorro, na estrada para a fazenda do Manso; 12.XII.1990, fr., H.C. de 4069 (photo RB!); Ponte Nova, Estrada BR-120, em direção a Ponte Nova, a 4 km da ponte sobre o Córrego Bom Jardim e a $8 \mathrm{~km}$ da ponte sobre o Ribeirão Vau Açu, 22.V.1978, f1., J.P. Fontella 1032 (photo RB!); Pedra Azul, 9.V.2003, I.R. Andrade et al. (BHCB 89476); Reduto, 3.VI.1954, fl., E.P. Heringer 3448 (UB); Rio Piracicaba, 23.VI.1989, H.C. Souza (BHCB 17158); Santana do Riacho, 12.VII.1987, I.R. Andrade \& M.B. Horta 149 (BHCB); São João Del Rei, 10.VI.2013, M. Sobral 15511 (BHCB); São Thomé das Letras, 30.IX.1998, fr., S.M. Faria 1683 (photo RB!); Teixeiras, estrada BR-120, direção a Ponte Nova, a 4 $\mathrm{km}$ da ponte sobre o córrego Bom Jardim e a $8 \mathrm{~km}$ da ponte sobre o ribeirão Vau-Açu, 22.V.1978, fl., Fontella 1032, M.R.R. Vidal 406, W.N. Vidal 376 (VIC); Teófilo Otoni, afloramento rochoso, lado esquerdo da MG 418, cerca de $30 \mathrm{~km}$ norte de Teófilo Otoni, 1.IV.2011, fl., L.F.A. de Paula et. al 339 (BHCB, photo RB!); Viçosa, 13.VIII.1979, fr., R.S. Ramalho e G. Rodrigues 1524 (IBGE), s.d., R.S. Ramalho 1348 (photo RB!), UFV, Silvicultura, s.d., R.S. Ramalho 1737 (photo RB!). 


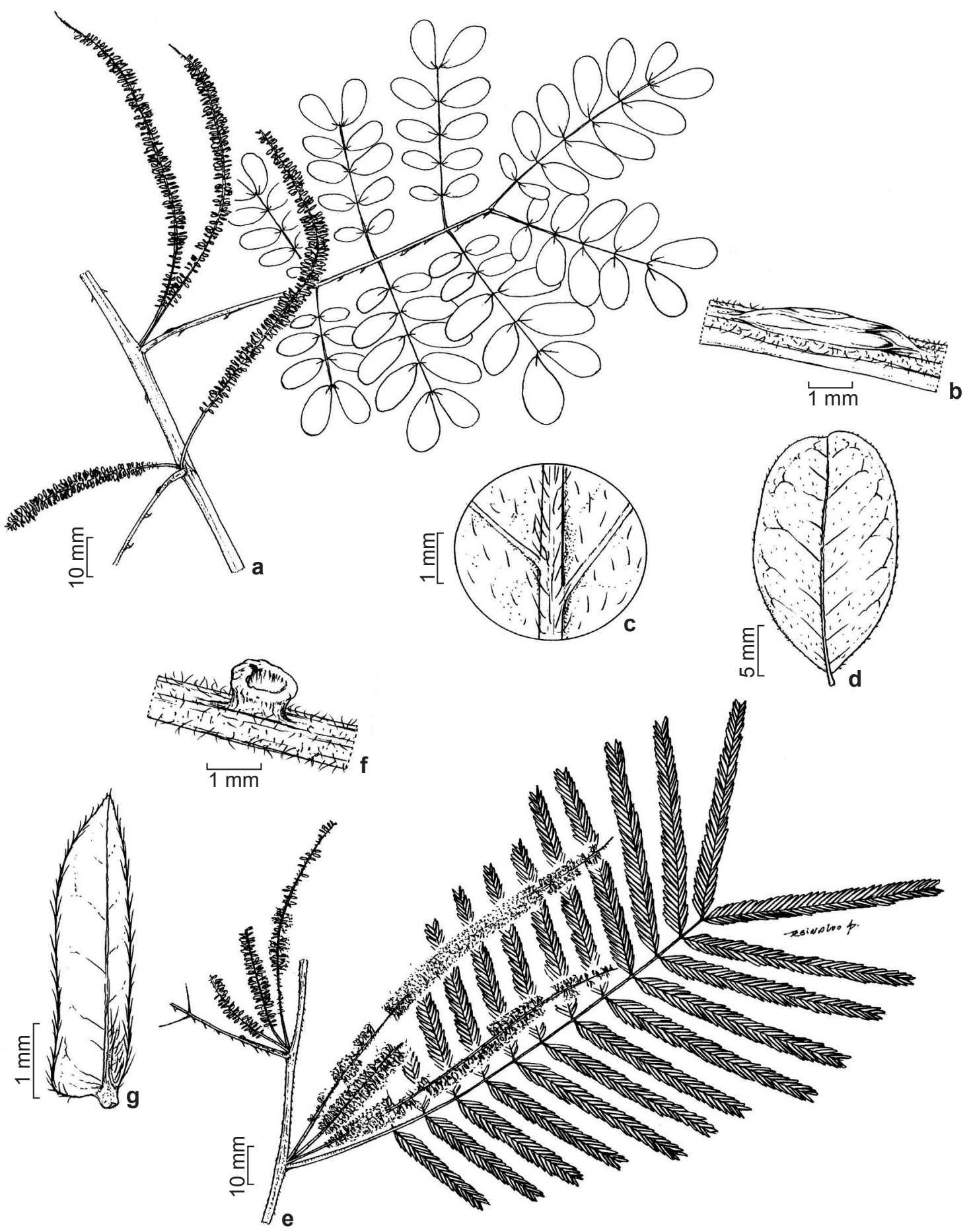

Figure 2 - a-d. Piptadenia adiantoides (Spreng.) J.F. Macbr. - a. branch with inflorescences; b. verruciform petiolar nectary; c. sericeous indumentum of the leaflets; d. leaflets (a,c,d L.C.P. Lima 310; b J.R. Stehmann 3076). e-g. Piptadenia gonoacantha (Mart.) J.F. Macbr. - e. branch with inflorescences; f. cupuliform petiolar nectary; g. leaflets (e,g R.L.C. Bortoluzzi 682; f D.S. Pifano 115). 
1.2. Piptadenia gonoacantha (Mart.) J.F. Macbr. Contributions from the Gray Herbarium of Harvard University 59: 17. 1919.

Figs. 2e-g; $4 \mathrm{~h}$

Tree, 4.5-20 m tall; trunk exfoliating; branches cylindrical with winged, armed, straight or curved aculeus, $0.6-2 \mathrm{~mm}$ long. Stipules deciduous, not observed. Leaves with 7-14 pairs of pinnae; leaf rachis 4-10 cm long, cylindrical, with straight or curved aculeus; leaflets linear, $27-50$ pairs per pinna, $5-8 \times 1-1.6 \mathrm{~mm}$, glabrous on both surfaces or sericeous on the abaxial surface with margin ciliated, base truncated, apex acute, eccentric midrib; petiolar nectary cupuliform. Inflorescences spiciform, 1-3 per axil, 6-9 cm long. Calyx, 0.7-0.9 mm long, sericeous; corolla $1.75-1.82 \mathrm{~mm}$ long, glabrous; ovary stipitate, glabrous. Legume, $10-16 \times 1.5-2.5 \mathrm{~cm}$; seed rounded, 6-9 × 6-8 mm.

Species restricted to South America in Brazil, Bolivia, Colombia and Peru (Oliveira-Filho 2006). In Brazil, it is nearly distributed throughout the entire country, occurring in Rio Grande do Norte, Paraíba, Pernambuco, Bahia, Alagoas, Sergipe, Mato Grosso do Sul, Minas Gerais, Espírito Santo, São Paulo, Rio de Janeiro, Paraná, Santa Catarina and Rio Grande do Sul (BFG 2015). In Minas Gerais, it was sampled in the south-central region (Fig. 3) in the Atlantic Forest and Cerrado domains (Oliveira-Filho 2006).

Piptadenia gonoacantha is mainly characterized by the presence of an exfoliating trunk and winged branches that allows to easily recognize the species even in a vegetative state. It differs from other species in Minas Gerais by also presenting a cupuliform petiolar nectary.

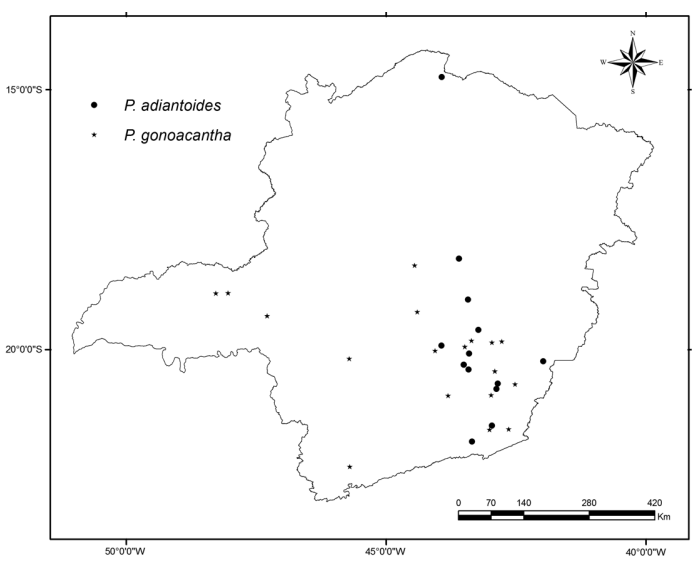

Figure 3-Distribution of Piptadenia adiantoides (Spreng.) J.F. Macbr. and P. gonoacantha (Mart.) J.F. Macbr. in Minas Gerais.
Examined material: Araponga, Comunidade São Joaquim, 5.IX.2005, fr., J.M. Fernandes 06 (VIC); Araxá, 19.XI.2010, J.R. Stehmann \& M. Augsten 6136 (BHCB); Barão de Cocais, Serra da Cambota, 2.VI.1992, est., M. Brandão 19.448 (PAMG); Belo Horizonte, FZB, Trilha do lobo, 16.I.2008, fl., J. Ordones et al. 222 (VIC); 9.V.2007, D.F. Felix 59 (BHCB); 3.I.1983, G.W.A. Fernandes \& E.Tameirão Neto (BHCB 13110); 1.I.2012, A. Portugal-Santana 14 (BHCB); 29.VIII.1990, E. Tameirão Neto 129 (BHCB); 19.IV.1991, E. Tameirão Neto \& C.Y.K. Matsuoka 389 (BHCB); 19.II.1991, E. Tameirão Neto (BHCB 23082); 1957, L.R. Rennó (BHCB 3614); 1982, T.S.M. Grandi \& R.C.F. Carvalho 112 (BHCB); 22.V.1942; T. Barbosa (BHCB 66425); Brumadinho, Inhotim, 29.IV.2008, fr., F.M. Rodrigues, F.M. (BHCB); 19.III.2008, F.M. Rodrigues \& J.G. Oliveira 219 (BHCB); 29.IV.2008, F.M. Rodrigues \& J.G. Oliveira 287 (BHCB); Carandái, Pedra do Sino Hotel Fazenda, BR 040, Km 6: Trilha da Mata atrás do hotel, 1.V.2005, fr., N.F.O. Mota \& P.L. Viana 220 (ICB, BHCB); Caparaó, Alto-Caparaó, propriedade Sr. Jorge Cortez, margem do rio Caparaó Braga, 3.I.1998, fl., J.M.A. 4702 (photo RB!); Caratinga, 18.III.1994, C.V. Mendonça 248 (BHCB); 25.IV.1994, C.V. Mendonça 279 (BHCB); 24.V.1984, P.M. de Andrade et al. 222 (BHCB); Conceição do Mato Dentro, Santo Antonio do Cruzeiro, Fazenda Eliezer Kamino, 2.VII.2008, fr., L.H.Y 1032 (BHCB, photo RB!); Corinto, 2.III.1970, fl., H.S. Irwin et al. 26763 (UB); Descoberto, Reserva Biológica do Grama, 7.V.2001, est., R.M. Castro et al. 322 (CESJ); Dionísio, Zona de amortecimento do PERD, Mata do Mombaça, 13.IV.2006, P.B. Souza et al. 19590 (VIC); Ibirité, Serra do Rola Moça, 21.III.1998, est., M. Brandão 28394 (PAMG); Iguatama, mata da estiva, 28.IV.2000, est., M. Brandão 29489 (PAMG); Juiz de Fora, 7.IX.2001, fr., D.S. Pifano \& M.O.D. Pivari 115 (CESJ); 11.I.2002, D.S. Pifano \& A.S.M. Valente 252 (BHCB); Leopoldina, Mata da Tulha, 25.V.1998, est., M. Brandão 28510 (PAMG); BR $116 \mathrm{Km}$ 757, fl., Hatschbach, G. 47669 (RB photo!); Mariana, Parque Estadual do Itacolomi, Trilha ao longo do rio Mainarte, 20.I.2005, est., L.C.P. Lima, F.C.P. Garcia \& M.E.F. de Araújo 264 (VIC); 2.XI.2000, R.C. da Mota \& L. Viana 628 (BHCB); 24.X.2002; A. Salino 8126 (BHCB); Manhuaçu, between Manhuaçu and Realeza, Km 4 E of junction of BR-116 and BR-262, 18.I.1985, fl., A.H. Gentry (photo RB!); Monte Carmelo, VII.2008, fr., I. Schiavini et al. 56051 (HUFU); IX.2008, I. Schiavini et al. 483 (BHCB); Nova Ponte, área 3, 11.VIII.1987, fr., J.R. Stehmann (photo RB 417742!); Nova Lima, IV.2005, A. Salino et al 10416 (BHCB); VIII.2005, A. Salino et al. 10578 (BHCB); Paraopeba, Fazenda do Barreiro, 29.III.1956, fl., E.P. Heringer 5146 (UB); Paula Candido, 2.I.2001, fl., E.W. Teixeira 25561 (VIC); Pedra Azul, próximo a Estiva Salim, 1.VII.1981, fr., J. Filho 170 (photo RB!); Perdizes, EPDA - Estação Ambiental Galheiro, 16.I.2003, fl., E.H. Amorim, S. Mendes, R. Arruda, D.V. Fonseca 425 (HUFU); Ponte 

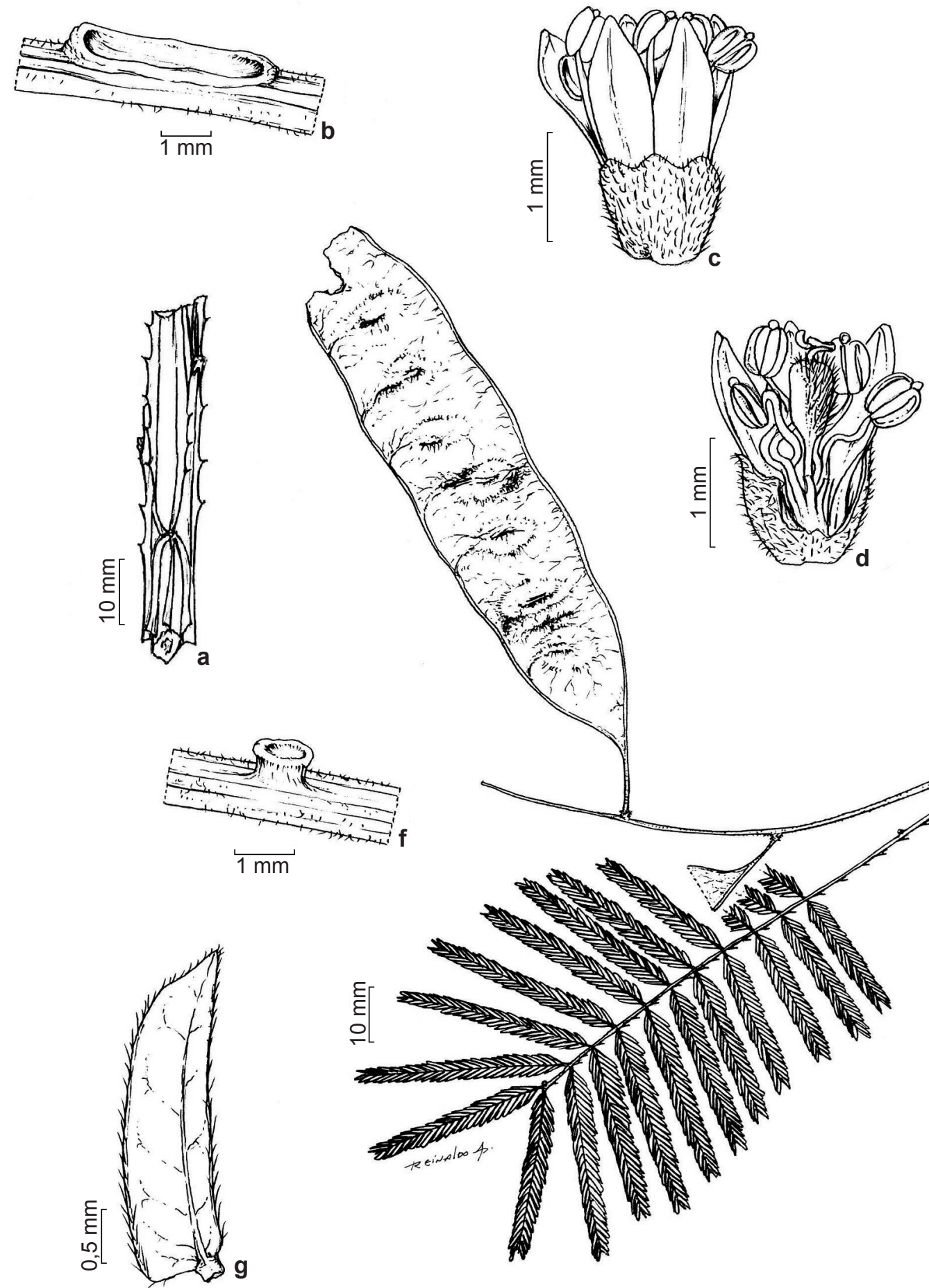

Figure 4 - a. Piptadenia gonoacantha (Mart.) J.F. Macbr. - detail of the winged branch. b-d. Piptadenia macradenia Benth. - b. patelliform petiolar nectary; c. flower; d. flower in longitudinal section. (J.R. Pirani et al. 3705). e-g. Piptadenia micracantha Benth. - e. branch with fruit; f. detail of the verruciform petiolar nectary and aculeus; g. leaflets. (e,g L.C.P. Lima 390; f R.S. Ramalho \& G. Rodrigues 1163). 
Nova, 16.II.1971, fl., J. Badini 4573 (VIC); Ribeirão das Neves, 7.XII.2009, N.C. Moreira et al. 110 (BHCB); Rio Vermelho, Fazenda Portão, 14.V.1986, fr., M.S. Menandro 78 (photo RB!); Santa Rita do Sapucai, 29.V.1994, M. Brandão 23964 (PAMG); São Domingos do Prata, Bacia do Macuco, 10.XI.1984, fr., M. Brandão 10966 (PAMG); São Gonçalo do Rio Abaixo, 2.X.1986, fr., Pedralli et al. 7524 (HXBH); São João Nepomuceno, Serra dos Núcleos - Fazenda Primavera, 11.II.2003, fl., D.S. Pifano et al. 458 (CESJ); São Miguel, 16.II.1930, fl., Y. Mexia 4359 (VIC). Uberlândia, Fazenda Buriti, 27.II.1994, fl., G.M. Araujo et al 1205 (VIC); Teófilo Otoni, 27.X.2011, fl., L.F.A. de Paula 608 (BHCB, photo RB!); 16.IV.2011, L.F.A. de Paula \& M. Augsten 311 (BHCB); 9.VII.1935, Mello Barreto1514 (BHCB); Vale do rio Araguari, Triângulo Mineiro, est., A.L.P. Mota et al. 4277 (VIC); Viçosa, 24.VIII.1999, fl., R.L.C. Bortuluzzi 682 (VIC); 8.VIII.1935, fr., J.G. Kuhlmann (photo RB 78313); 26.XII.1934, fl., J.G. Kuhlmann 1551 (photo RB!); 30.V.1978, fr., R.S. Ramalho 1177 (Photo RB!); 23.I.1983, fl., F.A. da Silveira (photo RB 235951); 26.III.1987, fl., A.L. Bernardi 2 (photo RB!).

1.3. Piptadenia macradenia Benth. Journal of Botany 4(30): 335-336. 1841.

Fig. 4i-k

Tree, ca. $10 \mathrm{~m}$ tall; branches cylindrical, unarmed. Stipules deciduous, not observed. Leaves with 7-9 pairs of pinnae; leaf rachis $5-7 \mathrm{~cm}$ long, cylindrical, unarmed; leaflets linear, 37-47 pairs per pinna, $7-8 \times 1.3-1.5 \mathrm{~mm}$, glabrous on the adaxial surface and sericeous on the abaxial surface, margin ciliated, apex acute, base truncate or cordate, midrib eccentric; petiolar nectary patelliform, oblong. Inflorescences spiciform, grouped in panicles, $6.3-8.5 \mathrm{~cm}$ long. Calyx, $0.9-1.1 \mathrm{~mm}$ long, tomentose; corolla $1.5-1.7 \mathrm{~mm}$ long, sericeous; ovary stipitate, villous in the upper half. Legume flat, $8.4-10 \times 1.8-2.4 \mathrm{~cm}$; seeds not observed.

Endemic to Brazil, where it is only found in the states of Goiás and Minas Gerais (Bentham 1875; BFG 2015). Species restricted to the Cerrado domain in the State of Minas Gerais (Fig. 6).

It resembles $P$. micracantha by presenting pinnae with a large number of leaflet pairs (32-50 pairs) but differs by presenting unarmed branches and patelliform petiolar nectaries.

Examined material: Paraopeba, Fazenda do Funil, 31.III.1960, fl., E.P. Heringer 7484 (UB); Santana do Riacho, Serra do Cipó, Morro da Pedreira, 2.IV.1996, fr., J.R. Pirani et al. 3705 (IBTSP); Estrada IBAMA - Distrito do Cipó (antigo Cardeal Mota), margem esquerda, em frente à chacara Vila Anstal, 18.III.2012, fl., L.M. Borges (photo RB 560455!); 25.II.1985, Andrade et al. (BHCB 8869); 14.IV.1985, Andrade et al. (BHCB 8913).
1.4. Piptadenia micracantha Benth. Transactions of the Linnean Society of London 30(3): 369. 1875.

Fig. 41-n

Scandent or climbers shrubs; branches cylindrical, armed, aculeus curved, 0.9-2 mm long. Stipules deciduous, not observed. Leaves 8-13 pairs of pinnae; leaf rachis 5-10 cm long, cylindrical, aculeate; leaflets linear, 32-50 pairs per pinna, 3-7 $\times 0.5-1 \mathrm{~mm}$, glabrous on both surfaces, rarely sericeous on the abaxial surface, margin ciliate, base truncated, apex acute or cuspidate, eccentric midrib; petiolar nectary verruciform, oblong. Inflorescences spiciform, 1-2 per axil, 3-7 cm long. Calyx, $0.9-1 \mathrm{~mm}$ long, glabrous; corolla 1.9-2 mm long, glabrous; ovary stipitate, tomentose. Legume 9-12 × 2.4-3.2 cm; seeds obovate, $6-8.5 \times 5.5-7 \mathrm{~mm}$.

Endemic to the southeast of Brazil, occurring in the states of Minas Gerais, São Paulo and Rio de Janeiro (Bentham 1875; BFG 2015). In Minas Gerais, it occurs in the Atlantic Forest domain (Fig. 6).

It is the only species that occurs solely in the Atlantic Forest and is easily recognized among other species of Piptadenia in Minas Gerais by its habit (scandent shrub or liana), the armed branches and the linear leaflets with $32-50$ pairs per pinna, which is the main character differentiating $P$. micracantha and $P$. adiantoides.

Examined material: Caratinga, Estação Biológica de Caratinga, Mata do Jaó, 23.IV.1984, fl., M.A. Lopes 331 (photo RB!); Caparaó, Serra do Caparaó, 30.IV.1988, fl., P.L. Krieger 079 (photo RB!); Lima Duarte, Ibitipoca, trilha atrás da casa da Polícia Florestal, 30.III.2004, fl., R.C. Forzza 3287 (CESJ); Manhuaçu, 2.VI.1954, fl., E.P. Heringer 3422 (UB); Mariana, Parque Estadual do Itacolomi, trilha ao longo do rio Mainarte, 23.VIII.2005, fr., L.C.P. Lima \& J.C. Duelhi-Filho 390 (VIC); Ouro Preto, Parque Estadual do Itacolomi, 14.IV.1994, est., J.L. Silva, M.C.T.B. Messias \& M.B. Roschel 20984 (VIC); São João Nepomuceno, Serra dos Núcleos, Fazenda Primavera, 24.II.2003, fr., A. Valente, R.O. Garcia et al. 295 (CESJ, BHCB); Tombos, 4.V.1941, fl., J.E. de Oliveira 327 (UB, BHCB); Viçosa, 19.V.1978, fl., R.S. Ramalho \& G. Rodrigues 1163 (IBGE).

1.5. Piptadenia paniculata Benth. Journal of Botany 4(31): 338. 1841.

Fig. 5o-t

Trees, 5-25 m tall; branches cylindrical, armed or unarmed; stipules deciduous, not observed. Leaves 3-7 pairs of pinnae; leaf rachis 4-12 cm long, cylindrical, unarmed; leaflets ovallanceolate, $7-15$ pairs per pinna, $15-30 \times 7-13$ $\mathrm{mm}$, sericeous on both surfaces, base obtuse, apex 


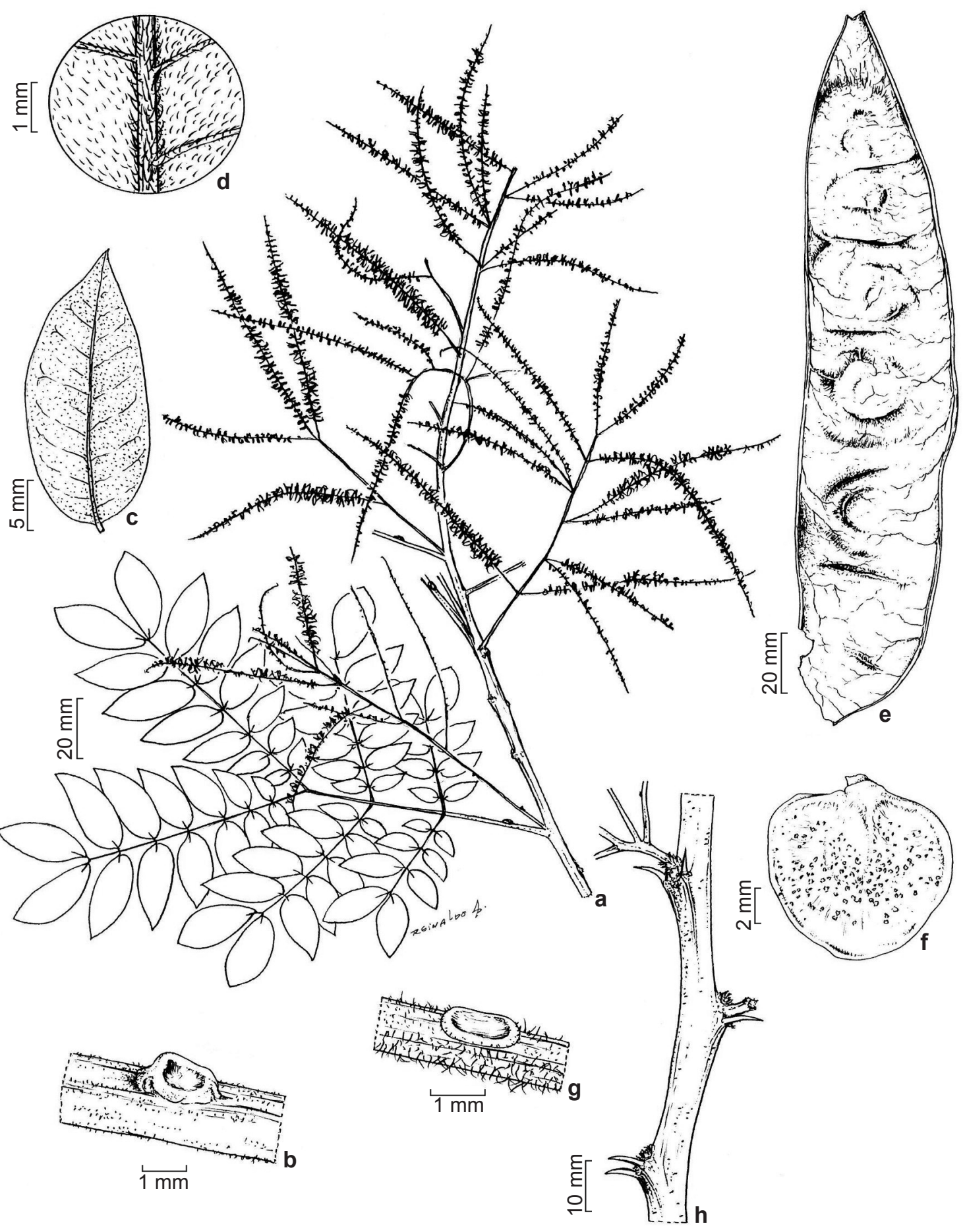

Figure 5 - a-f. Piptadenia paniculata Benth. - a. branch with inflorescences; b. detail of the patelliform petiolar nectary; c. leaflets; d. detail of the sericeous indumentum of the leaflets; e. legume; f. suborbicular seed (a $F$. da Silva 119; c,d J. de Castro 2195-B; b W.F. Lopes 17092). g-h. Piptadenia viridiflora (Kunth.) Benth. - g. detail of the patelliform petiolar nectary; h. stipules modified into spines and paired at the nodes (J.M. Fernandes 1185). 
acute, mucronate, central midrib; petiolar nectary patelliform. Inflorescences spiciform, grouped in panicles, 6-18 cm long. Calyx 0.5-0.7 $\mathrm{mm}$ long, sericeous; corolla 1.7-2 mm long, glabrous or sericeous; ovary stipitate, tomentose. Legume $8-20 \times 1.7-4.6 \mathrm{~cm}$; seed suborbicular to oblong, $1.1-1.3 \times 1.2-1.3 \mathrm{~cm}$.

Endemic to Brazil, it is primarily distributed in the northeast, southeast and south regions in the states of Bahia, Minas Gerais, Espírito Santo, São Paulo, Rio de Janeiro, Paraná and Santa Catarina (Oliveira-Filho 2006; BFG 2015). In Minas Gerais, it is found in the southeast and west regions (Fig. 6), occurring in Cerrado and Atlantic Forest vegetation.

When compared with other species of the genus, it is easily recognizable due to the longer shape of the leaflets with more than two pairs of pinnae (Ribeiro 2012). Piptadenia paniculata is similar to $P$. adiantoides by the size of the leaflets, but the former has obovate-elliptical or ovate leaflets, and the latter has oval-lanceolate leaflets. Another detail is the shape of the petiolar nectary, which is verruciform-oblong in $P$. adiantoides and patelliform in $P$. paniculata. Examined material: Bias Fortes, 6.V.1952, fr., L. Roth 1895 (photo RB!); Conceição das Alagoas, Represa de Volta Grande, 5.X.1988, fr., G. Pedralli \& J.R. Stehmann 5505 (HXBH); Coronel Pacheco, 28.I.1952, fl. e fr., E.P. Heringer 2579 (UB); Descoberto, Reserva Biológica da Represa do Grama, fl., L. Meireles 2001 (photo RB!); Juiz de Fora, VII.1945, fl., P.L. Krieger 1093 (CESJ); Morro do Imperador, 8.IV.2003, fr., D.S. Pifano 521 (photo RB!), 26.II.1969, fl., V. Gomes (photo RB 144181!); Ouro Preto, 14.XII.1990, fl., H.C. de Lima 4113 (photo RB!); Teixeiras, 26.II.1969, fr., E.P. Heringer 1361 (UB); Viçosa, 15.VII.1935, fr., J. de Castro 2195-B (VIC), fl., 13.VIII.1979, fr., R.S. Ramalho 1524 (photo RB!), 27.XII.1978, fr., R.S. Ramalho 1348 (photo RB!), 1.II.1983, fl., R.S. Ramalho 2549 (photo RB!), fl., G.L. Rodrigues (photo RB 173841!); Tombos, 18.VII.1935, H.L. Mello Barreto 1655 (BHCB).

1.6. Piptadenia viridiflora (Kunth) Benth. Journal of Botany 4(31): 337. 1841. Fig. 5u-v

Trees up to $7 \mathrm{~m}$ tall; trunk smooth; branches cylindrical. Stipules modified into aculeus paired at the nodes. Leaves $11-15$ pairs of pinnae; leaf rachis 6-12 cm long, cylindrical, unarmed; leaflets linear, 24-34 pairs per pinnae, 4-6.5 $\times 1-2 \mathrm{~mm}$, sericeous on both surfaces, base truncate, apex acute, eccentric midrib; petiolar nectary patelliform. Inflorescences speciform, two or three per axil, $6.3-8.8 \mathrm{~cm}$ long. Flowers not observed. Legume flat, compressed, 10.5-19 $\times 1.7-2.5 \mathrm{~cm}$. Seeds were not observed.

Species restricted to South America in Argentina, Brazil and Bolivia. In Brazil, occurs in the states of Ceará, Paraíba, Pernambuco, Bahia, Mato Grosso do Sul and Minas Gerais (Oliveira-Filho 2006; BFG 2015). In Minas Gerais, it is found in the north and south regions of the state in the three vegetation domains (Fig. 6).

Piptadenia viridiflora is easily recognized, even when sterile, due to its significant, paired nodal aculeus representing modified stipules (spiny) (Ribeiro 2012). The green and speciform inflorescences, which are characters unique in the genus, also differentiate it from the other species of the genus (Ribeiro 2012). Seeds were not observed because the fruits were collected in the early stage of maturation.

Examined material: Águas Vermelhas, 6.V.1979, fr., E.C. Tenório 79 (photo RB!); Espinosa, rio Capivara, 1.XI.1991, fr., G. Pedralli 25 (photo RB!); Itacarambi, 15.V.2010, C. Vidal \& T. Mansur 825 (BHCB); Itacambira, 29.I.2010, E. Tameirão NetoVidal 4794 (BHCB); Januária, 30.VI.1976, fr., J.A. Ratter, J. Fonseca Filho, S.G. da Fonseca 3236 (UB); 29.VII.1993, E. Tameirão Neto 914 (BHCB); 21.VII.1997, A. Salino \& J.R. Stehmann 3306 (BHCB); Juiz de Fora, Mata da Candeia, 4.IV.2003, fr., W.C. Carvalho et al. 41891 (CESJ); Manga, 18.I.2010, fr., J.M. Fernandes 1215 (VIC); 30.IV.1991, T.C.S. Sposito 64 (BHCB); Fazenda dos Portugueses, 26.VI.1974, fr., M. Magalhães 160 (photo RB!); Matias Cardoso, Parque Estadual Verde Grande, 18.II.2009, fr., J.M. Fernandes 1021 (VIC); Montes Claros, 13.XI.2010, fr., J.M. Fernandes 1185 (VIC).

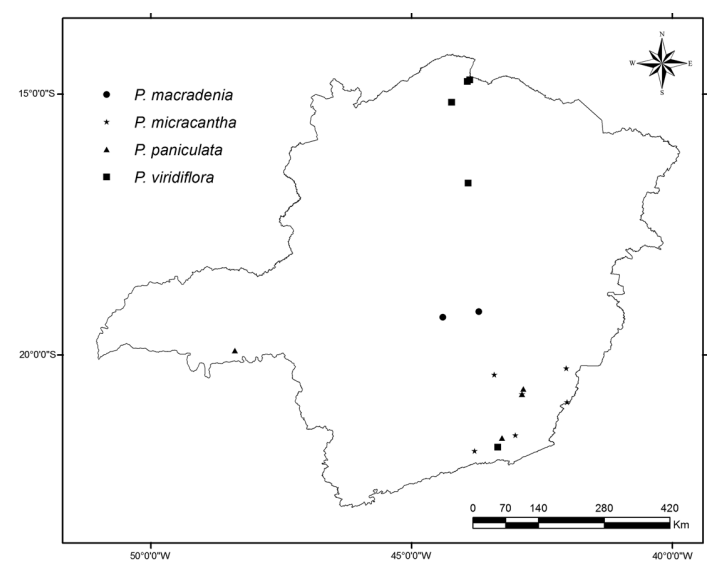

Figure 6 - Distribution of Piptadenia macradenia Benth., P. micracantha Benth., P. paniculata Benth. and $P$. viridiflora (Kunth.) Benth. in Minas Gerais. 
Species not confirmed in Minas Gerais

Although Oliveira-Filho 2006 reported the occurrence of Piptadenia irwinii G.P. Lewis and $P$. stipulacea (Benth.) Ducke in Minas Gerais, these species were not found in the herbaria collections or in our field work. Queiroz (2009) and Morim (2010) stated that the former is restricted to Bahia state, and the latter is endemic to the Caatinga areas in the Brazilian northeast. Another species described in the literature for the state (OliveiraFilho 2006) but not mentioned in the present study is $P$. moniliformis Benth., which was transferred to the genus Pityrocarpa (Jobson \& Luckow 2007).

\section{Conclusion}

The state of Minas Gerais proved to be rich in the number of species of Piptadenia, accounting for approximately $29 \%$ of the diversity of this genus in Brazil. All species are native, and most are endemic to Brazil. Only $P$. gonoacanth $a$ and $P$. viridiflora occur in other South American countries. In Minas Gerais, the genus is represented in the three phytogeographic domains: Atlantic Forest, Cerrado and Caatinga, but only $P$. adiantoides and $P$. viridiflora are found in all three domains. $P$. macradenia is restricted to the Cerrado and $P$. micracantha to the Atlantic Forest, where, with five species, the highest diversity was found.

In most species, the flowering period usually extends from December to April with fruiting from April to August, but it may last until October in some species.

\section{Acknowledgments}

We would like to acknowledge all of the herbaria that kindly provided specimens as well as the Brazilian agency CNPq for the scholarship granted to the first author and FAPEMIG for its financial support. We would also like to thank Reinaldo Pinto for the beautiful illustrations.

\section{References}

Ab'Sáber, A.N. 2003. Os domínios de natureza no Brasil: potencialidades paisagísticas. Ateliê Editorial, São Paulo. 144p.

Bentham, G. 1840. Contributions towards a Flora of South América. Enumeration of plants collectes by Mr. Schomburgk in British Guiana. Journal of Botany 2: 127-147.

Bentham, G. 1875. Revision of the suborder Mimoseae. Transactions of the Linnean Society of London 30: 335-668.
BFG. 2015. Growing knowledge: an overview of seed plant diversity in Brazil. Rodriguésia 66: 1085-1113.

Drummond, G.M.; Martins, C.S.; Machado, A.B.M.; Sebaio, F.A. \& Antonini, Y. 2005. Biodiversidade em Minas Gerais: um atlas para sua conservação. Fundação Biodiversitas, Belo Horizonte. 222p.

Grimes, J.W. 1999. Inflorescence morphology, heterochrony, and phylogeny in the Mimosoid tribes Ingeae and Acacieae (Leguminosae: Mimosoideae). Botanical Review 65: 317-347.

Fernandes, J.M. 2011. Ingeae Benth. (Leguminosae, Mimosoideae) no estado de Minas Gerais, Brasil: taxonomia, morfoanatomia de nectários extraflorais aplicada à evolução, distribuição geográfica, fitogeografia, uso e conservação. Tese de Doutorado. Universidade Federal de Viçosa, Viçosa. 294p.

Holmgren, P.K. \& Holmgren, N.H. 2009. Index Herbariorum. Available at $<$ http://sweetgum.nybg. org/ih/>. Access on July $1^{\text {st }} 2013$.

IBGE. 1992. Manual técnico da vegetação brasileira. IBGE, Rio de Janeiro. 92p.

Jobson, R.W. \& Luckow, M. 2007. Phylogenetic study of the genus Piptadenia (Mimosoideae: Leguminosae) using plastid trnL-F and trnk-matK sequence data. Systematic Botany 32: 569-575.

Lewis, G.P.; Schrire, B.D.; Mackinder, B.A. \& Lock, J.M. 2005. Legumes of the world. Royal Botanic Gardens, Kew. 577p.

Luckow, M.; Miller, J.T.; Murphy, D.J. \& Livshultz, T. 2003. A phylogenetic analysis of the Mimosoideae (Leguminosae) based on choroplast DNA sequence data. In: Klitgaard, B.B. \& Bruneau, A. (eds.). Advances in legume systematics, part 10, higher level systematics. Royal Botanic Gardens, Kew. Pp. 197-220.

Luckow, M. 2005. Tribe Mimoseae. In: Lewis, G.P.; Schrire, B.D.; Mackinder, B. \& Lock, M. (eds.). Legumes of the world. Royal Botanic Gardens, Kew. Pp. 162-183.

MMA - Ministério do Meio Ambiente. 2008. Lista oficial de espécies da flora brasileira ameaçada de extinção. Diário Oficial da União de 24 de setembro de 2008 185, 1: 75-83.

Morim, M.P. 2010. Piptadenia Benth. In: Forzza RC (org.). Catálogo de plantas e fungos do Brasil. Vol. 2. Instituto Jardim Botânico do Rio de Janeiro, Rio de Janeiro. Pp. 1077-1078.

Oliveira-Filho, A.T. 2006. Catálogo das árvores nativas de Minas Gerais: mapeamento e inventário da flora nativa e dos reflorestamentos de Minas Gerais. Ed. UFLA, Lavras. 423p.

Queiroz, L.P. 2009. Leguminosas da Caatinga. Universidade Estadual de Feira de Santana, Feira de Santana. 467p.

Reflora- Herbário Virtual. Available at <http:// www.herbariovirtualreflora.jbrj.gov.br/reflora/ herbarioVirtual/>. Access on March 2016. 
Ribeiro, P.G. 2012. Flora da Bahia: família Leguminosae, sub-família Mimosoideae: Tribo Acacieae \& Tribo Mimoseae - Parte 1. Dissertação de Mestrado. Universidade Estadual de Feira de Santana, Feira de Santana. 371p.

Tamashiro, J.Y. 1989. Estudos taxonômicos e morfológicos do gênero Piptadenia, sensu Bentham no sudeste do Brasil. Avaliação das modificações taxonômicas recentemente propostas. Dissertação de Mestrado. Universidade Estadual de Campinas, Campinas. 99p.

Veloso, H.P.; Rangel-Filho, A.L.R. \& Lima, J.C.A. 1991. Classificação da vegetação brasileira, adaptada a um sistema universal. Rio de Janeiro, IBGE. 112p. 